\title{
Heat Transfer on Magneto hydrodynamic Peristaltic Flow in a Porous Medium with Partial Slip
}

\author{
Mohammed Salim * Ahmed M. Abd_alhadi \\ Department of mathematics, university of Baghdad, Iraq
}

\begin{abstract}
The present study concerned with the impact of velocity slip on MHD peristaltic flow through a porous medium with heat transfer is investigated. The relevant equations of flow with heat transfer have been developed. Analytic solution is carried out under long-wavelength and small Reynolds number approximations. The expressions for the stream function, temperature and the heat transfer coefficient are obtained. Numerical results are graphically discussed for various values of physical parameters of interest.
\end{abstract}

Keywords: Peristalsis, MHD, porous medium, slip flow, compliant walls.

\section{Introduction}

The transportation of many biological fluids is carried out with the help of naturally inherited mechanism inside the biological systems which is called peristalsis. It is nature's way of moving the content within hollow muscular structures by successive contraction of their muscular fibers. This principle is responsible for transport of biological fluids such as urine in the ureter, chime in the gastrointestinal tract, semen in the vas deferens, ovum in the fallopian tube, lymph transport in the lymphatic vessels, blood pumps in the heart lung machine etc. In plant physiology, the peristalsis is involved in phloem translocation by driving a sucrose solution along tubules by peristaltic contractions. The corrosive and noxious fluids can also be transported by peristalsis. Such flows in presence of heat transfer also have great value. This process is useful for the analysis of tissues, oxygenation and dialysis. Roller and finger pumps also work under the peristaltic mechanism. The seminal research on the peristaltic motion has been presented by Latham [1] and Jaffrin and Shapiro [2]. Since then the various experimental and theoretical studies have been presented in the viscous and non-Newtonian fluids [3-6]. In view of the importance of oxygenation and dialysis, the peristaltic flows with heat transfer have been also investigated [7-10]. The influence of heat and mass transfer on MHD peristaltic flow through a porous space with compliant walls was investigated by Srinivas et al. [11].

In several applications the flow pattern corresponds to a slip flow, and the fluid presents a loss of adhesion at the wetted wall making the fluid slide along the wall. when the molecular mean free path length of the fluid is comparable to the distance between the plates as in nanochannels or microchannels, the fluid exhibits non-continuum effects such as slip-flow as demonstrated experimentally by Derek et al. [12]. Investigations of the effects of slip on the peristaltic motion have been recently reported in [13-14].

The aim of the present paper is to discuss the velocity slip effect on the MHD peristaltic transport of non-Newtonian fluid in a porous space with heat transfer. Such an analysis is of great interest in bio-medical research. The momentum, temperature equations have been linearized under long wavelength and low Reynolds number assumption and exact solutions for the flow fluid dynamical variables have been derived. The contribution of several interesting parameters embedded in the flow system is examined by graphical representations.

\section{The mathematical formulation of the problem}

The motion of heat and mass transfer peristaltic flow of a Newtonian viscous fluid through a twodimensional channel of uniform thickness filled with porous medium is considered. The motion in the channel is induced by imposing moderate amplitude sinusoidal waves on the compliant wall of the channel as shown in figure (2.1) and thus the wall are defined by

$$
\bar{y}= \pm \bar{\eta}(\bar{x}, \bar{t})= \pm\left[d+a \sin \frac{2 \pi}{\lambda}(\bar{x}-c \bar{t})+\bar{m} \bar{x}\right]
$$

Where $d$ is the mean half width of the channel, $a$ is the amplitude, $\lambda$ is the wave length, $\bar{t}$ is the time, $\bar{m}$ is the non-uniform parameter and $c$ is the phase speed of the wave respectively

The magnetic Reynolds number and induced magnetic field are assumed to be small and neglected. Under these assumptions the governing equation of continuity, momentum, heat transfer are as follow: 

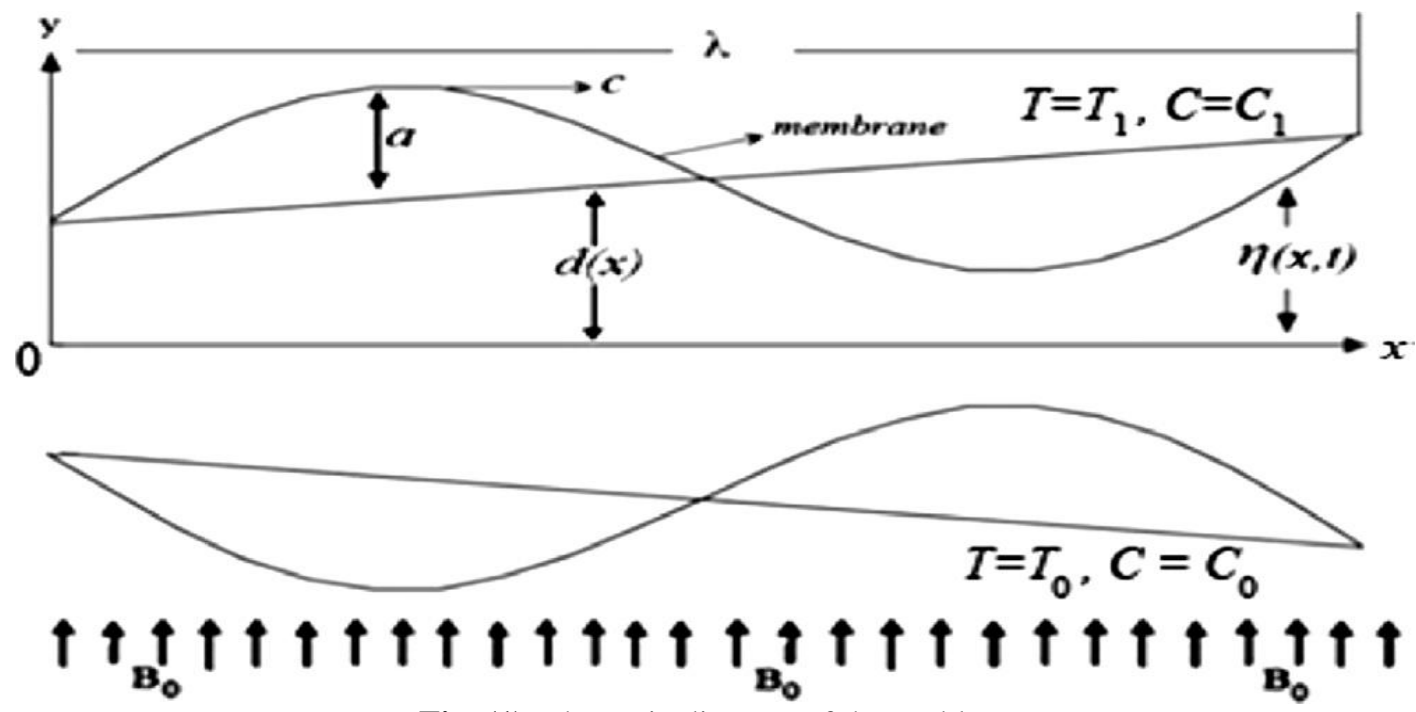

Fig. (1) schematic diagram of the problem

$$
\begin{aligned}
& \frac{\partial \bar{u}}{\partial \bar{x}}+\frac{\partial \bar{v}}{\partial \bar{y}}=0 \\
& \rho\left(\frac{\partial \bar{u}}{\partial \bar{t}}+\bar{u} \frac{\partial \bar{u}}{\partial \bar{x}}+\bar{v} \frac{\partial \bar{u}}{\partial \bar{y}}\right)=-\frac{\partial \bar{p}}{\partial \bar{x}}+\mu\left[\frac{\partial^{2} \bar{u}}{\partial \bar{x}^{2}}+\frac{\partial^{2} \bar{u}}{\partial \bar{y}^{2}}\right]-\sigma B_{0}^{2} \bar{u}-\frac{\mu}{k} \bar{u} \\
& \rho\left(\frac{\partial \bar{v}}{\partial \bar{t}}+\bar{u} \frac{\partial \bar{v}}{\partial \bar{x}}+\bar{v} \frac{\partial \bar{v}}{\partial \bar{y}}\right)=-\frac{\partial \bar{p}}{\partial \bar{y}}+\mu\left[\frac{\partial^{2} \bar{v}}{\partial \bar{x}^{2}}+\frac{\partial^{2} \bar{v}}{\partial \bar{y}^{2}}\right]-\frac{\mu}{k} \bar{u} \\
& \zeta\left(\frac{\partial T}{\partial \bar{t}}+\bar{u} \frac{\partial T}{\partial \bar{x}}+\bar{v} \frac{\partial T}{\partial \bar{y}}\right)=\frac{\underline{g}}{\rho}\left[\frac{\partial^{2} T}{\partial \bar{x}^{2}}+\frac{\partial^{2} T}{\partial \bar{y}^{2}}\right]+v_{1}\left[\left(\frac{\partial \bar{u}}{\partial \bar{y}}+\frac{\partial \bar{v}}{\partial \bar{x}}\right)^{2}+2\left(\left(\frac{\partial \bar{u}}{\partial \bar{y}}\right)^{2}+\left(\frac{\partial \bar{v}}{\partial \bar{x}}\right)^{2}\right)\right]
\end{aligned}
$$

Where $\bar{u}, \bar{v}$ are the components of velocity along $\mathrm{x}$ and $\mathrm{y}$ direction, $\bar{p}$ is the pressure, $\mu$ is the coefficient of viscosity of the fluid, $\beta$ is the coefficient of thermal expansion, $\sigma$ is the electrical conductivity of the fluid, $k$ is the permeability parameter, $B_{0}$ is the applied magnetic field, $\alpha$ is the thermal diffusivity, $v_{1}$ is the kinematic viscosity, $\rho$ is the density of the fluid, $\zeta$ is the specific heat at constant pressure, $\xi_{\mathrm{r}}$ is the chemical reaction of rate constant and $T$ is the temperature.

The governing equation of motion of the flexible wall is expressed as

$$
L^{*}(\bar{\eta})=\bar{p}-\bar{p}_{0}
$$

Where $L^{*}$ is an operator, which is used to represent the motion of stretching membrane with viscosity damping forces such that

$$
L^{*}=-\tau \frac{\partial^{2}}{\partial \bar{x}^{2}}+m \frac{\partial^{2}}{\partial \bar{t}^{2}}+C \frac{\partial}{\partial \bar{t}}+B \frac{\partial^{4}}{\partial \bar{x}^{4}}+H
$$

Here $\tau$ is the elastic tension in the membrane, $m$ is the mass per unit area, $C$ is the coefficient of viscous damping forces, $B$ is the flexural rigidity of the plate, $H$ is the spring stiffness and $p_{0}$ is the pressure on the outside surface of the wall due to the tension in the muscles and assume that $\bar{p}_{0}=0$.

The associated boundary condition for the velocity slip and temperature at the wall interface are given by

$$
\bar{u}=\mp \beta^{*} \frac{\partial \bar{u}}{\partial \bar{y}}, \quad T=T_{0} \quad \text { at } \bar{y}= \pm \bar{\eta}= \pm\left[d+a \sin \frac{2 \pi}{\lambda}(\bar{x}-c \bar{t})+\bar{m} \bar{x}\right]
$$

And the boundary conditions due to wall flexibility are

$$
\begin{aligned}
& \frac{\partial}{\partial \bar{x}} L^{*}(\bar{\eta})=\frac{\partial \bar{p}}{\partial \bar{x}} \\
&=\mu\left[\frac{\partial^{2} \bar{u}}{\partial \bar{x}^{2}}+\frac{\partial^{2} \bar{u}}{\partial \bar{y}^{2}}\right]-\rho\left[\frac{\partial \bar{u}}{\partial \bar{t}}+\bar{u} \frac{\partial \bar{u}}{\partial \bar{x}}+\bar{v} \frac{\partial \bar{u}}{\partial \bar{y}}\right]-\sigma B_{0}^{2} \bar{u}-\frac{\mu}{k} \bar{u} \quad \text { at } \bar{y}= \pm \bar{\eta}
\end{aligned}
$$




\section{Dimensionless analysis}

To make the Equations (2)-(9) non-dimensional, it is convenient to introduce the following non-dimensional variables and parameters:

$x=\frac{\bar{x}}{\lambda}, \quad y=\frac{\bar{y}}{\lambda}, \quad t=\frac{c \bar{t}}{\lambda}, \quad \eta=\frac{\bar{\eta}}{d}, \quad p=\frac{d^{2} \bar{p}}{c \lambda \mu}, \quad \theta=\frac{T-T_{0}}{T_{0}}, \quad \epsilon=\frac{a}{d}, \quad \delta=\frac{d}{\lambda}, \quad R=\frac{\rho c d}{\mu}, \quad M=\sqrt{\frac{\sigma}{\mu} B_{0} d}$, $K=\frac{k}{d^{2}}, \quad \operatorname{Pr}=\frac{\rho v_{1} \zeta}{k}, \quad E c=\frac{c^{2}}{\zeta T_{0}}, \quad \beta=\frac{\beta^{*}}{d}, \quad E_{1}=\frac{-\tau d^{3}}{\lambda^{3} \mu c}, \quad E_{2}=\frac{m c d^{3}}{\lambda^{3} \mu}, \quad E_{3}=\frac{c d^{3}}{\lambda^{2} \mu}, \quad E_{4}=\frac{B d^{3}}{c \mu \lambda^{5}}, \quad E_{5}=\frac{H d^{3}}{\lambda c \mu}$, $m=\frac{\bar{m} \lambda}{d}, \quad v_{1}=\frac{\mu}{\rho}$

Where $R, \delta, M, K, P r, E c, \beta$ and $E_{1}, E_{2}, E_{3}, E_{4}, E_{5}$ are the Reynolds number, wave number, Hartmann number, permeability parameter, Prandtl number, Eckert number, Velocity slip parameter and wall compliant parameters.

Introducing the stream function $\psi\left(u=\frac{\partial \psi}{\partial y}, v=-\frac{\partial \psi}{\partial x}\right)$, equations (2)-(9) can be writing as:

$\delta R\left[\frac{\partial^{2} \psi}{\partial t \partial y}+\frac{\partial \psi}{\partial y} \frac{\partial^{2} \psi}{\partial x \partial y}-\frac{\partial \psi}{\partial x} \frac{\partial^{2} \psi}{\partial y^{2}}\right]=-\frac{\partial p}{\partial x}+\delta^{2} \frac{\partial^{3} \psi}{\partial x^{2} \partial y}+\frac{\partial^{3} \psi}{\partial y^{3}}-M^{2} \frac{\partial \psi}{\partial y}-\frac{1}{K} \frac{\partial \psi}{\partial y}$

$\delta^{3} R\left(-\frac{\partial \psi}{\partial t \partial x}-\frac{\partial \psi}{\partial y} \frac{\partial^{2} \psi}{\partial x^{2}}+\frac{\partial \psi}{\partial x} \frac{\partial^{2} \psi}{\partial y \partial x}\right)=-\frac{\partial p}{\partial y}+\delta^{2}\left[-\delta^{2} \frac{\partial^{3} \psi}{\partial x^{3}}-\frac{\partial^{3} \psi}{\partial y^{2} \partial x}\right]-\frac{\delta}{K} \frac{\partial \psi}{\partial y}$

$\delta R\left(\frac{\partial \theta}{\partial t}+\frac{\partial \psi}{\partial y} \frac{\partial \theta}{\partial x}-\frac{\partial \psi}{\partial x} \frac{\partial \theta}{\partial y}\right)=\frac{1}{P r}\left[\delta^{2} \frac{\partial^{2} \theta}{\partial x^{2}}+\frac{\partial^{2} \theta}{\partial y^{2}}\right]+E c\left[\left(\frac{\partial^{2} \psi}{\partial y^{2}}-\delta^{2} \frac{\partial^{2} \psi}{\partial x^{2}}\right)^{2}+4 \delta^{2}\left(\frac{\partial^{2} \psi}{\partial x y}\right)^{2}\right]$

$$
\frac{\partial \psi}{\partial y}=\mp \beta \frac{\partial^{2} \psi}{\partial y^{2}}, \quad \theta=0 \quad \text { at } y= \pm \eta= \pm[1+\epsilon \sin 2 \pi(x-t)+m x]
$$

$\left[E_{1} \frac{\partial^{3}}{\partial x^{3}}+E_{2} \frac{\partial^{3}}{\partial t^{2} \partial x}+E_{3} \frac{\partial^{2}}{\partial t \partial x}+E_{4} \frac{\partial^{5}}{\partial x^{5}}+E_{5} \frac{\partial}{\partial x}\right](\eta)=\left[\delta^{2} \frac{\partial^{3} \psi}{\partial x^{2} \partial y}+\frac{\partial^{3} \psi}{\partial y^{3}}\right]-\delta R\left[\frac{\partial^{2} \psi}{\partial y \partial t}+\frac{\partial \psi}{\partial y} \frac{\partial^{2} \psi}{\partial x \partial y}-\frac{\partial \psi}{\partial x} \frac{\partial^{2} \psi}{\partial y^{2}}\right]-$ $M^{2} \frac{\partial \psi}{\partial y}-\frac{1}{K} \frac{\partial \psi}{\partial y} \quad$ at $y= \pm \eta$

With the assumption of long wavelength approximation and neglecting the wave number $(\delta \ll 1)$ and small Reynolds number in equation (11)-(13) along with the boundary condition (14) and (15), we get

$-\frac{\partial p}{\partial x}+\frac{\partial^{3} \psi}{\partial y^{3}}-N^{2} \frac{\partial \psi}{\partial y}=0$

$-\frac{\partial p}{\partial y}=0$

$\frac{\partial^{2} \theta}{\partial y^{2}}+B r\left(\frac{\partial^{2} \psi}{\partial y^{2}}\right)^{2}$

The corresponding dimensionless boundary conditions are

$$
\begin{gathered}
\frac{\partial \psi}{\partial y}=\mp \beta \frac{\partial^{2} \psi}{\partial y^{2}}, \quad \theta=0 \quad \text { at } y= \pm \eta= \pm[1+\epsilon \sin 2 \pi(x-t)+m x] \\
\frac{\partial^{3} \psi}{\partial y^{3}}-N^{2} \frac{\partial \psi}{\partial y}=\left[E_{1} \frac{\partial^{3}}{\partial x^{3}}+E_{2} \frac{\partial^{3}}{\partial t^{2} \partial x}+E_{3} \frac{\partial^{2}}{\partial t \partial x}+E_{4} \frac{\partial^{5}}{\partial x^{5}}+E_{5} \frac{\partial}{\partial x}\right](\eta) \\
= \pm \eta
\end{gathered}
$$

$= \pm \eta$
Here $N^{2}=M^{2}+\frac{1}{K}$ and $B r=\operatorname{Pr} E c$ is the Brinkman number.

\section{Solution of the problem}

The set of equations (16)-(18) are solved exactly for expression $\psi$ and $\theta$ and it's found that:

$$
\begin{aligned}
\psi & =\frac{e^{N y} c 1}{N^{2}}+\frac{e^{-N y} c 2}{N^{2}}+c 3+y c 4 \\
\theta & =-\frac{B r\left(c 2^{2} e^{-2 N y}+c 1^{2} e^{2 N y}+4 c 1 c 2 N^{2} y^{2}\right)}{4 N^{2}}+b 1+y b 2
\end{aligned}
$$

The velocity can be written as:

$u=\frac{\partial \psi}{\partial y}=c 4-\frac{c 2 e^{N y}}{N}+\frac{c 1 e^{N y}}{N}$

By the boundary conditions (19) and (20), we can find the constant value c1, c2, c3, c4, b1, b2 which are: $c 1=-\left(e^{N(-1-m x-\epsilon \operatorname{Sin}[2 \pi(-t+x)])}\left(E 5 m+2 E 5 \pi \epsilon \operatorname{Cos}[2 \pi(-t+x)]-8 E 1 \pi^{3} \epsilon \operatorname{Cos}[2 \pi(-t+x)]\right.\right.$

$$
\begin{aligned}
& -8 E 2 \pi^{3} \epsilon \operatorname{Cos}[2 \pi(-t+x)]+32 E 4 \pi^{5} \epsilon \operatorname{Cos}[2 \pi(-t+x)]+4 E 3 \pi^{2} \epsilon \operatorname{Sin}[2 \pi(-t \\
& +x)])) /\left(N\left(-1-e^{2 N(-1-m x-\epsilon \operatorname{Sin}[2 \pi(-t+x)])}-N \beta+e^{2 N(-1-m x-\epsilon \operatorname{Sin}[2 \beta(-t+x)])} N \beta\right)\right)
\end{aligned}
$$




$$
\begin{aligned}
& c 2=-\left(e ^ { N ( - 1 - m x - \epsilon \operatorname { S i n } [ 2 \pi ( - t + x ) ] ) } \left(E 5 m+2 E 5 \pi \epsilon \operatorname{Cos}[2 \pi(-t+x)]-8 E 1 \pi^{3} \epsilon \operatorname{Cos}[2 \pi(-t+x)]\right.\right. \\
& -8 E 2 \pi^{3} \epsilon \operatorname{Cos}[2 \pi(-t+x)]+32 E 4 \pi^{5} \epsilon \operatorname{Cos}[2 \pi(-t+x)]+4 E 3 \pi^{2} \epsilon \operatorname{Sin}[2 \pi(-t \\
& c 3=0 \\
& +x)])) /\left(N\left(1+e^{2 N(1+m x+\epsilon \operatorname{Sin}[2 \pi(-t+x)])} N \beta\right)\right) \\
& c 4=\left(8 E 1 \pi^{3} \epsilon \operatorname{Cos}[2 \pi(-t+x)]+8 E 2 \pi^{3} \epsilon \operatorname{Cos}[2 \pi(-t+x)]-32 E 4 \pi^{5} \epsilon \operatorname{Cos}[2 \pi(-t+x)]\right. \\
& \left.-E 5(m+2 \pi \epsilon \operatorname{Cos}[2 \pi \pi(-t+x)])-4 E 3 \pi^{2} \epsilon \operatorname{Sin}[2 \pi(-t+x)]\right) / N 2 \\
& b 1=-(1 /(2+2 m x+2 \epsilon \operatorname{Sin}[2 \pi(-t+x)]))(-((B r(1+m x+\epsilon \operatorname{Sin}[2 \pi(-t \\
& +x)])\left(c 2^{2} e^{2 N(-1-m x-\epsilon \operatorname{Sin}[2 \pi(-t+x)])}+c 1^{2} e^{2 N(-1-m x-\epsilon \operatorname{Sin}[2 \pi(-t+x)])}\right. \\
& \left.\left.\left.+4 c 1 c 2 N^{2}(-1-m x-\epsilon \operatorname{Sin}[2 \pi(-t+x)]) 2\right)\right) /\left(4 N^{2}\right)\right)+(B r(-1-m x \\
& -\epsilon \operatorname{Sin}[2 \pi(-t+x)])\left(c 2^{2} e^{-2 N(1+m x+\epsilon \operatorname{Sin}[2 \pi(-t+x)])}+c 1^{2} e^{2 N(-1-m x-\epsilon \operatorname{Sin}[2 \pi(-t+x)])}\right. \\
& +4 c 1 c 2 N 2(1+m x+\epsilon \operatorname{Sin}[2 \beta(-t+x)]) 2)) /(4 N 2)) \\
& b 2=-\left(\left(\operatorname{Br}\left(-c 1^{2}+c 2^{2}\right) e^{-2 N(1+m x+\epsilon \operatorname{Sin}[2 \pi(-t+x)])}\left(-1+e^{-4 N(1+m x+\epsilon \operatorname{Sin}[2 \pi(-t+x)])}\right)\right) /(8 N 2(1+m x\right. \\
& +\epsilon \operatorname{Sin}[2 \pi(-t+x)])))
\end{aligned}
$$

All the solutions in this section are made by using MATHEMATICA package.

\section{Result and discussion}

The purpose of this section is to study the influences of various parameters on the velocity, temperature and stream line (see figures (2)-(33)).

In the present study following default parameters value are adopted for computations: $E_{1}=1, E_{2}=0.5, E_{3}=$ $0.5, E_{4}=0.2, E_{5}=0.1, K=0.1, B r=0.2, \quad \beta=0.1, \quad M=1, m=0.1, t=0.1, \epsilon=0.1$ and $x=(0.4$ in velocity, 0.2 in temperature and stream line). All graphs therefore correspond to these values unless specifically indicated on the appropriate graph.

All the results in this section are made through plotting by using MATHEMATICA package.

\section{(5.1) stream line:}

The formation of internally circulating bolus of fluid through closed stream line is called trapping and this trapped bolus is pushed ahead along with the peristaltic wave. The effect of $M, K, \beta, E_{1}, E_{2}, E_{3}, E_{4}$, $E_{5}, m, t, \epsilon$ can be seen through figures (2)-(8) respectively. It reveals that the volume of the trapped bolus deceases with increase Hartmann number $M$. Notice that the stream line closed loops creating a cellular flow pattern in the channel and trapped bolus increases in size as permeability parameter $K$ increases. The size of the trapping bolus increase with increasing slip parameter $\beta$. The effect of wall complaint parameters obtain as the size of trapped bolus increases by increasing $E_{3}, E_{4}, E_{5}$ while it decrease with increasing $E_{1}, E_{2}$. Also the size of the trapping bolus increases with increasing non-uniform parameter $m$, time $t$ and $\epsilon$.

\section{(5.2) Variation of temperature}

The temperature profiles for various values of $M, \beta, x, K, m, B r, \epsilon, t, E_{1}, E_{2}, E_{3}, E_{4}$,

$E_{5}$ can be seen through figures (9)-(21) respectively. We observe that the temperature decreases with increasing Hartmann number $M$, velocity slip parameter $\beta$, and $x$ while the opposite behavior for the permeability parameter $K$, non-uniform parameter $m$, Brinkman number Brand $\epsilon$. The temperature increases with increasing time $t$ then it decreases. The effect of wall complaint parameters obtain as the temperature increases by increasing $E_{3}, E_{4}$ while it decrease with increasing $E_{1}, E_{2}$ and it not change with change $E_{5}$.

\section{(5.3) Variation of velocity}

The influence of $M, K, t, \beta, m, x, \epsilon, E_{1}, E_{2}, E_{3}, E_{4}, E_{5}$ on the velocity distribution can see graphically in figures (22)-(33) respectively. It reveals that increasing Hartmann number $M$, leads to fall in the velocity because the effect of increasing in magnetic field strength dampens the velocity. The effect of permeability parameter $K$ that when it increasing the velocity leads to enhance. An increasing $K$ means reduce the drag force and hence cause the flow velocity to increase. Also the velocity decreases with increasing time $t$. The velocity increases with increasing velocity slip parameter $\beta$, non-uniform parameter $m, x$ and $\epsilon$. The effect of wall compliant parameters that obtain as the velocity increases with increasing $E_{4}$ and decreases with increasing $E_{1}, E_{2}, E_{3}$ But the velocity not change with change $E_{5}$ 
(a)

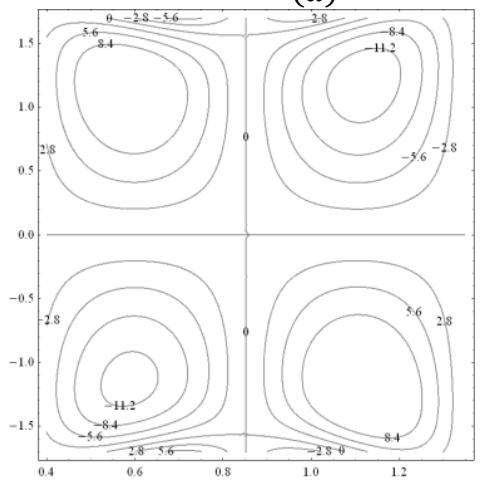

(b)

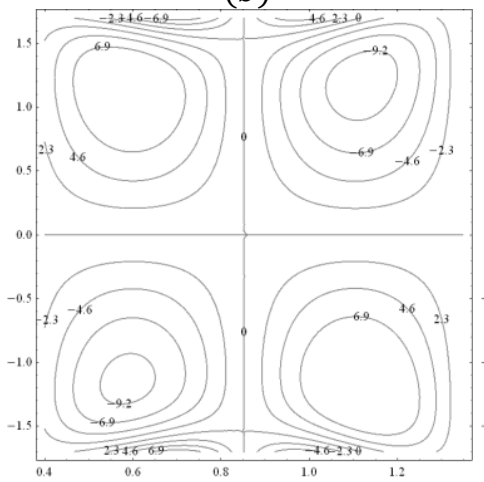

Fig. (2) Stream line for (a) $M=1$, (b) $M=2$, (C) $M=3$

(c)

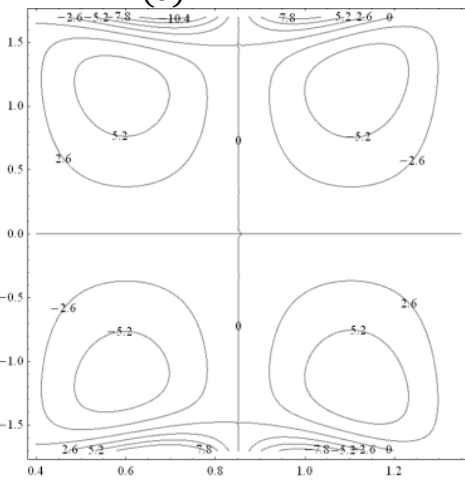

(a)

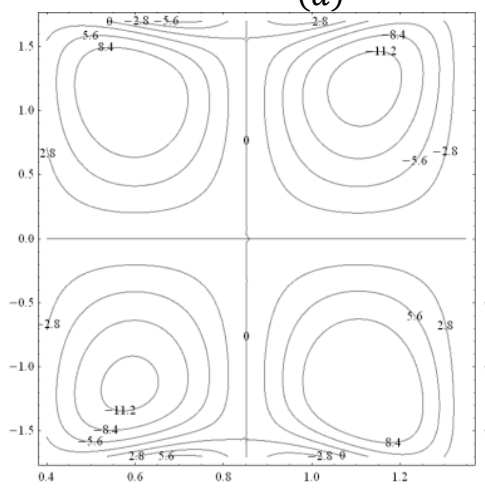

(b)

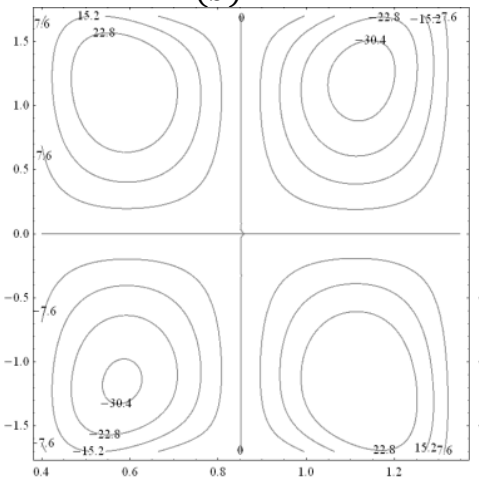

(c)

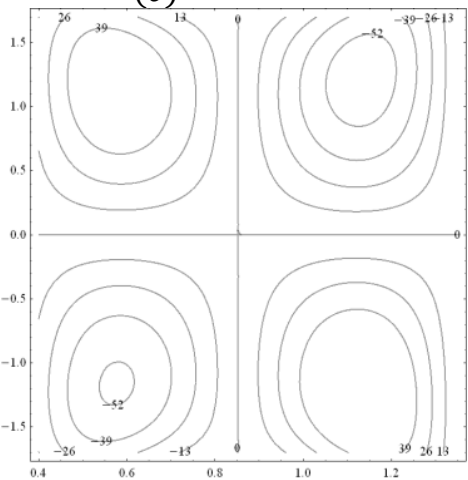

Fig. (3) Stream line for (a) $K=0.1$, (b) $K=0.5$, (C) $K=\infty$

(a)

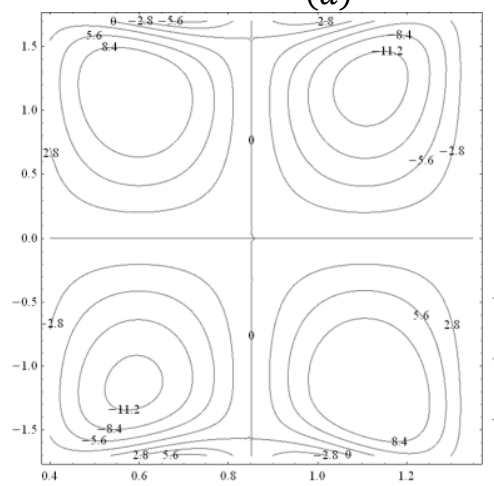

Fig. (4) Stream line for (a) $\beta=0.1$, (b) $\beta=0.2$, (c) $\beta=0.3$
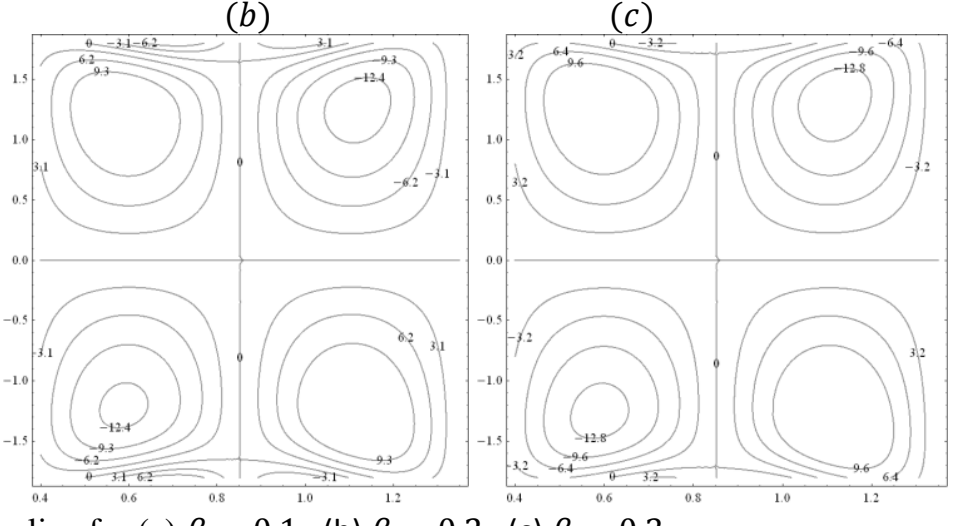

(a)

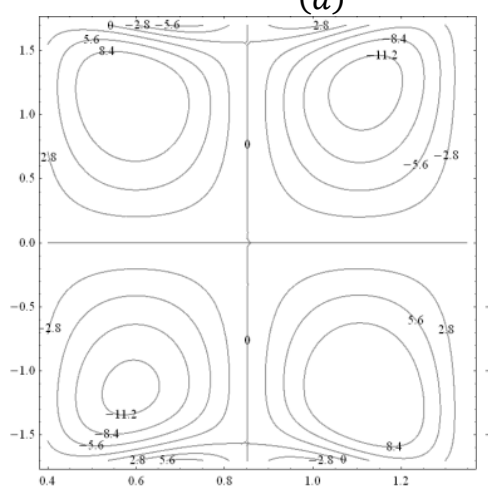

(b)

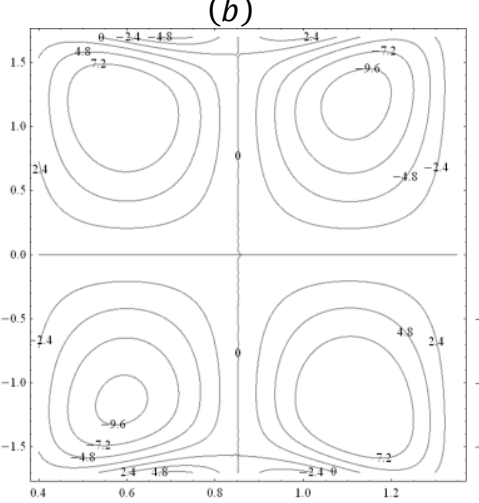

(c)

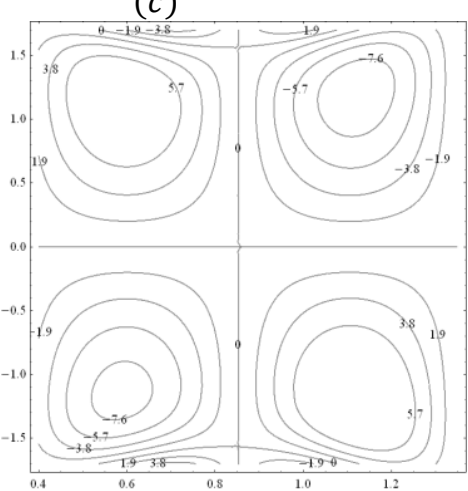


$(d)$

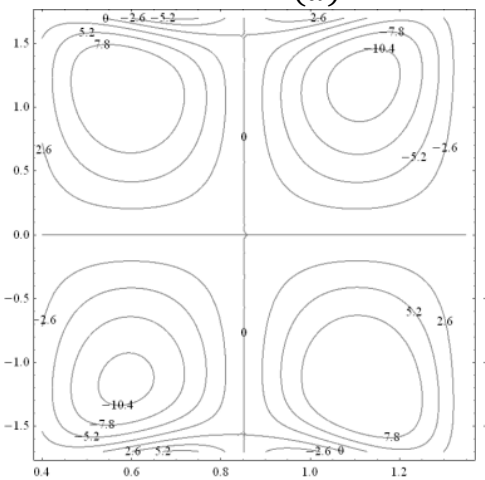

$(g)$

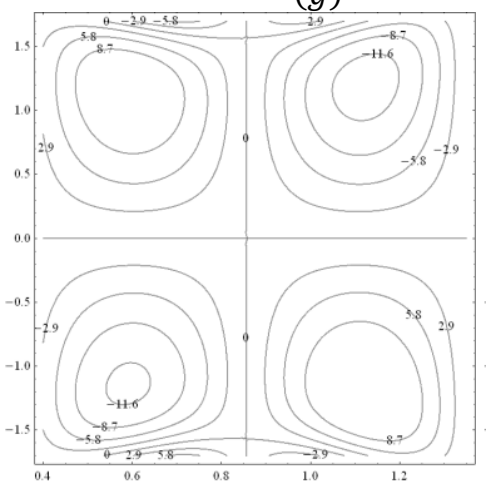

(e)

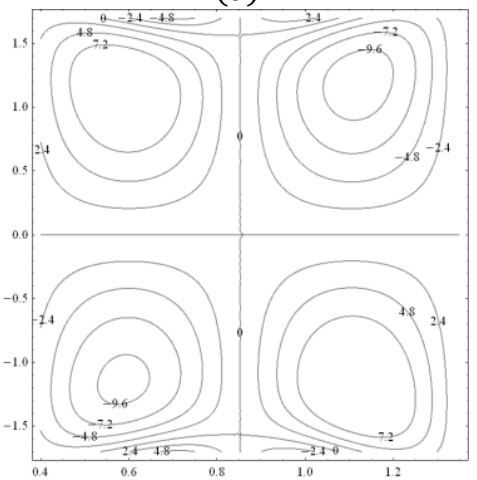

(h)

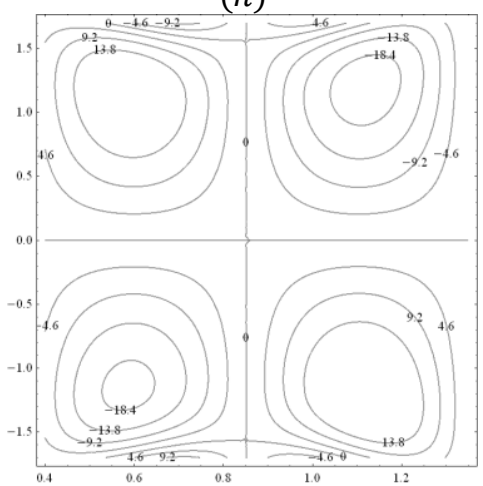

$(f)$

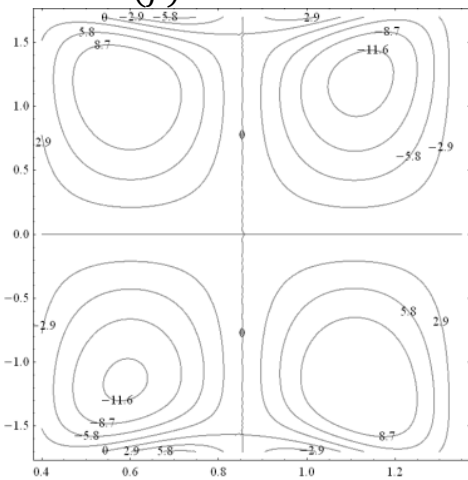

(i)

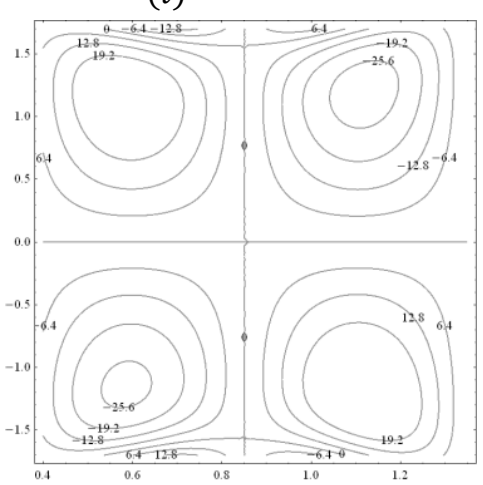

$(k)$

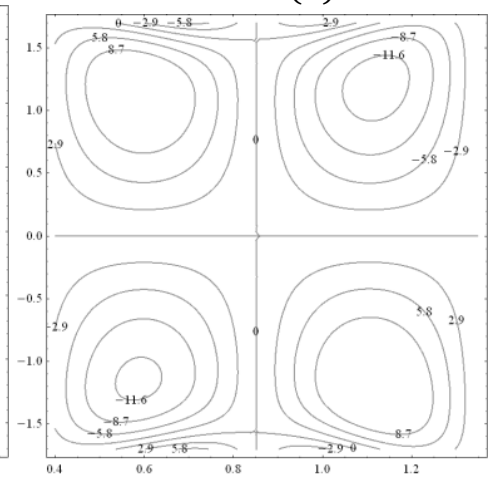

Fig. (5) Stream line for (a) $E_{1}=1,(b) E_{1}=2,(c) E_{1}=3,(d) E_{2}=1,(e) E_{2}=1.5,(f) E_{3}=1,(g) E_{3}=$ 1.5, $(h) E_{4}=0.3,(i) E_{4}=0.4,(j) E_{5}=0.5,(k) E_{5}=1$

(a)

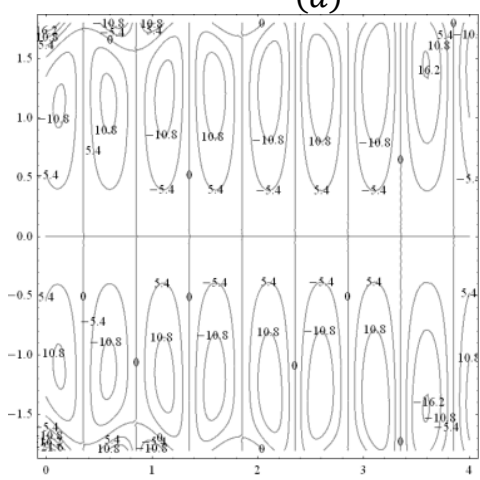

Fig. (6) Stream line for (a) $m=0.1$, (b) $m=0.3$, (c) $m=0.5$ (c)

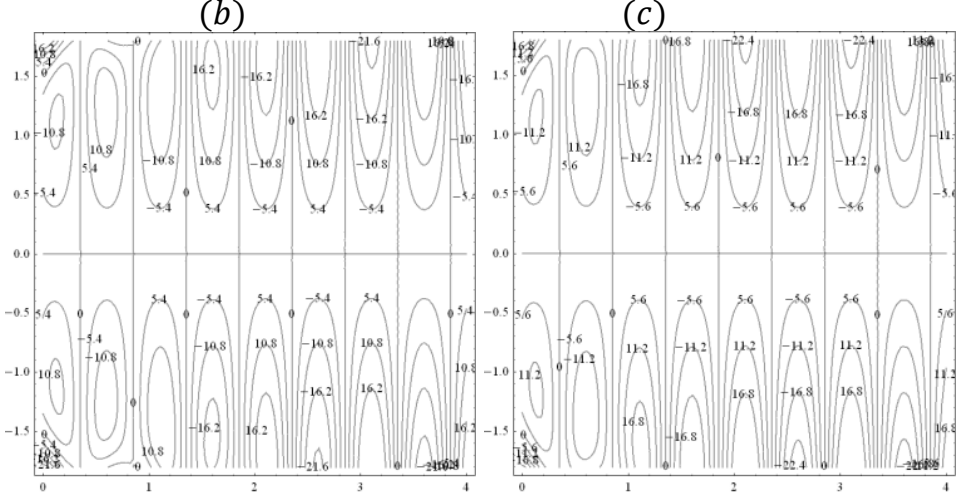


(a)

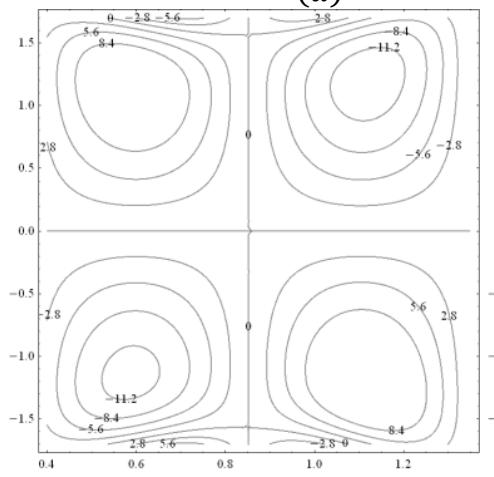

(b)

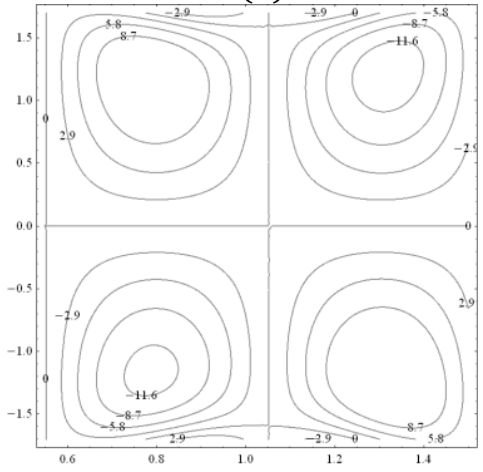

(c)

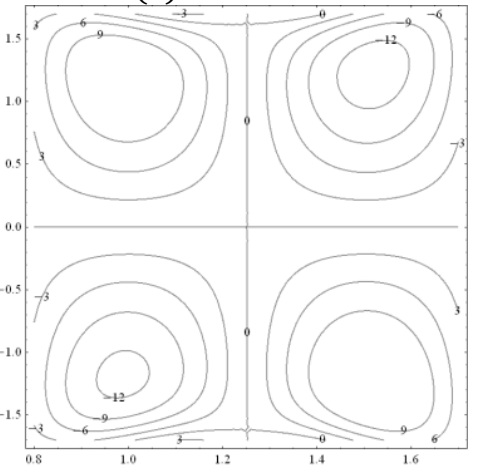

Fig. (7) Stream line for (a) $t=0.1,(b) t=0.3,(c) t=0.5$

(a)

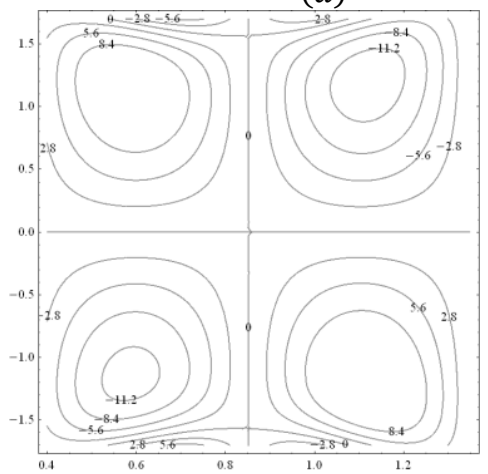

(b)

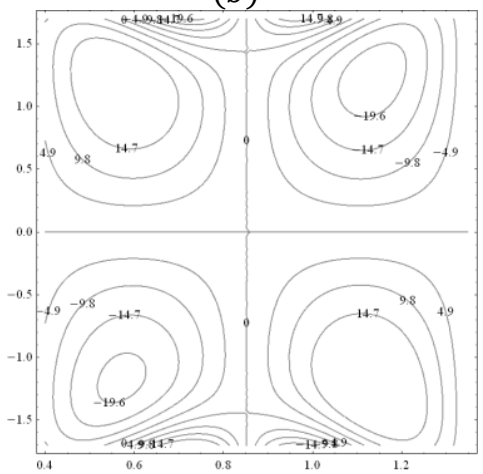

(c)

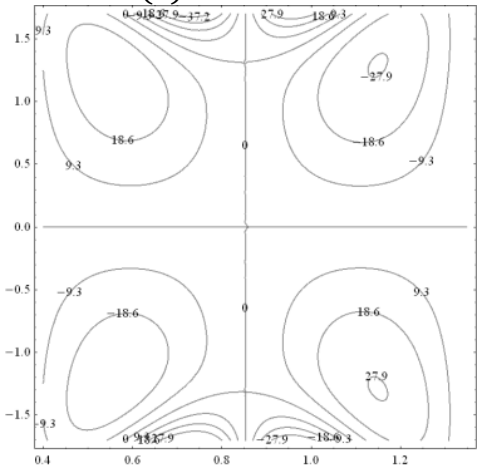

Figs. (8) Stream line for $(a) \epsilon=0.1,(b) \epsilon=0.2,(c) \epsilon=0.3$

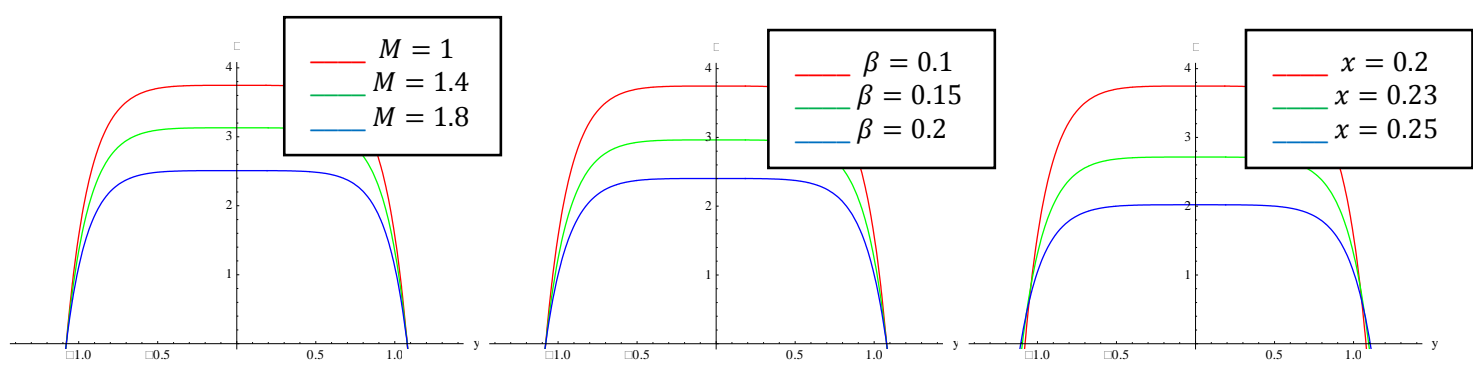

Figs. (9), (10), (11) variation of temperature $\theta$ for different value of $M, \beta, x$ respectively

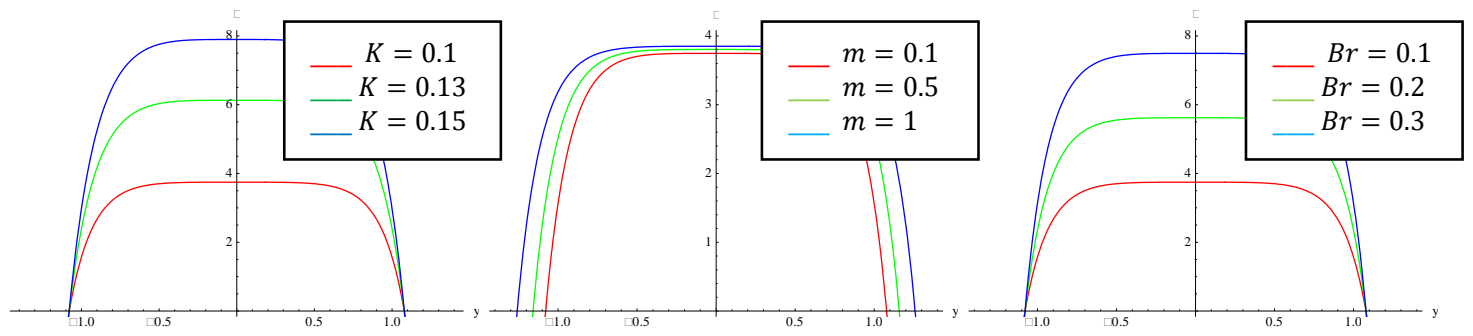

Figs. (12), (13), (14) variation of temperature $\theta$ for different value of $K, m, B r$ respectively 


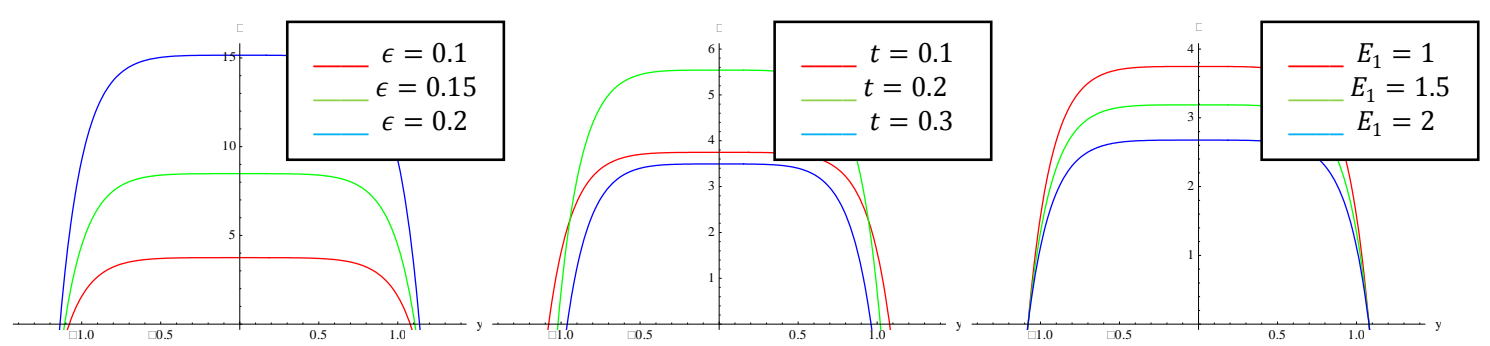

Figs. (15), (16), (17) variation of temperature $\theta$ for different value of $\epsilon, t, E_{1}$ respectively

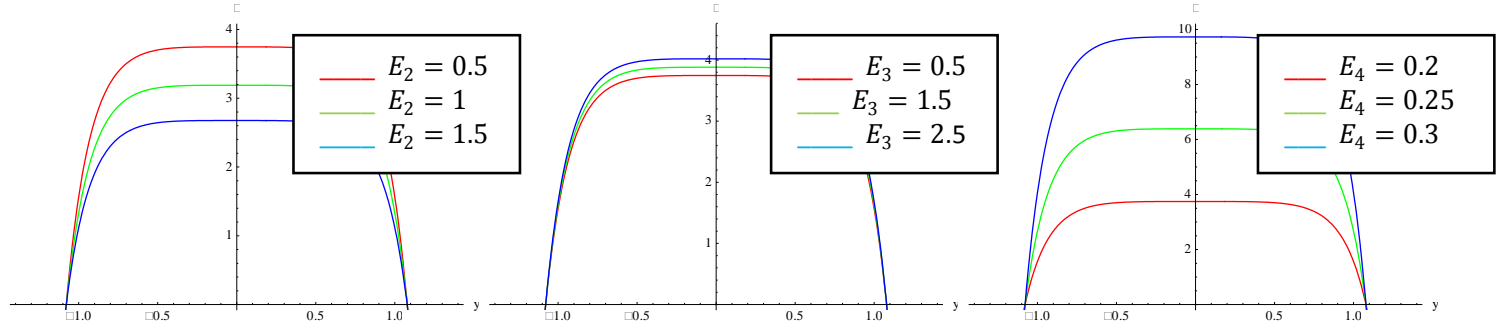

Figs. (18), (19), (20) variation of temperature $\theta$ for different value of $E_{2}, E_{3}, E_{4}$ respectively

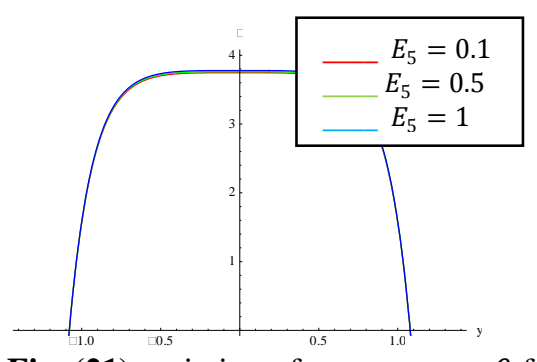

Fig. (21) variation of temperature $\theta$ for different value of $E_{5}$ different value of $M$

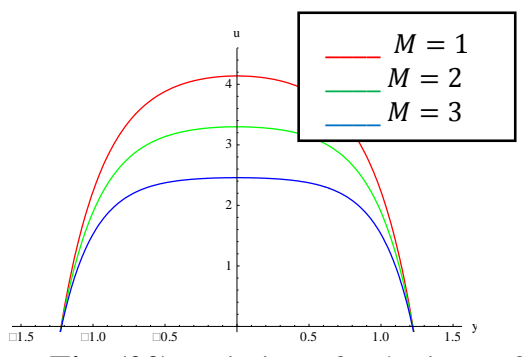

Fig. (22) variation of velocity $u$ for

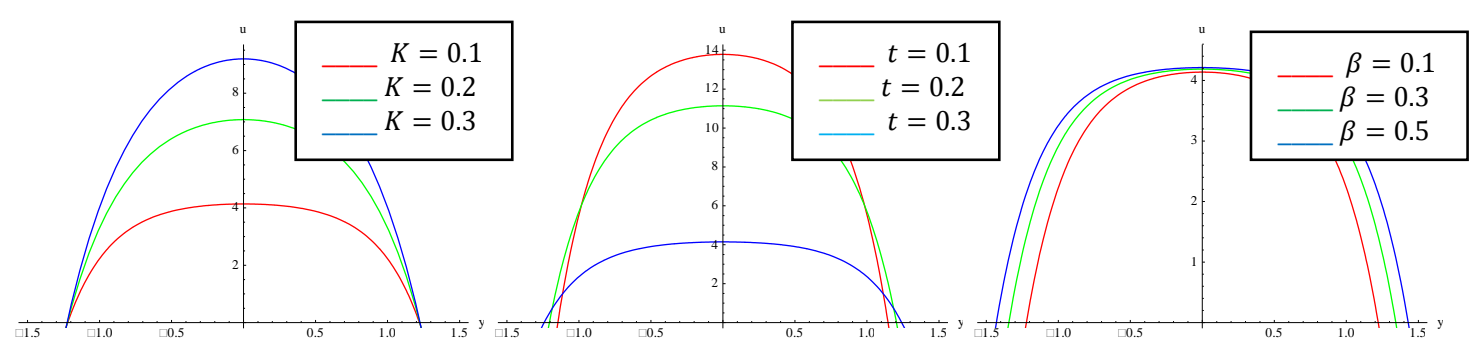

Figs. (23), (24), (25) variation of velocity $u$ for different value of $K, t, \beta$ respectively

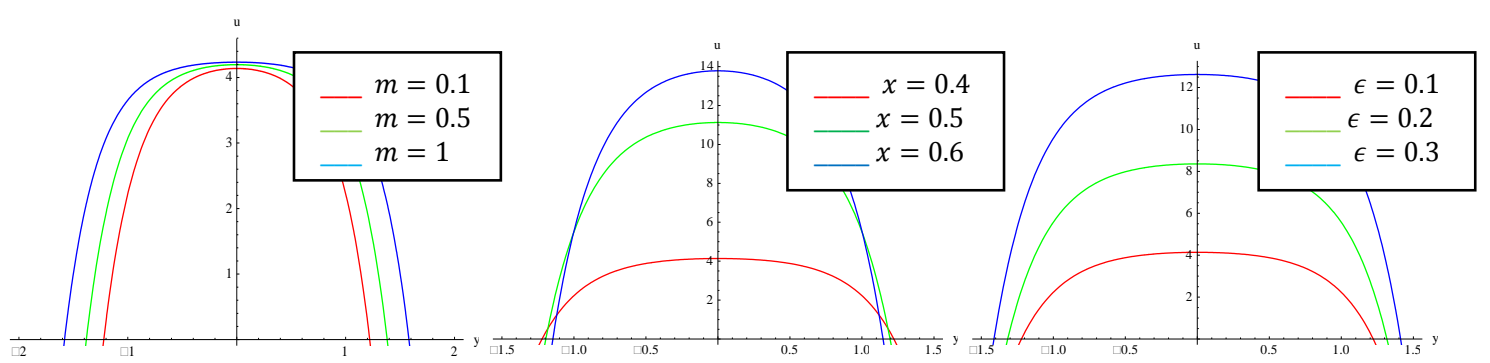

Figs. (26), (27), (28) variation of velocity $u$ for different value of $m, x, \epsilon$ respectively 


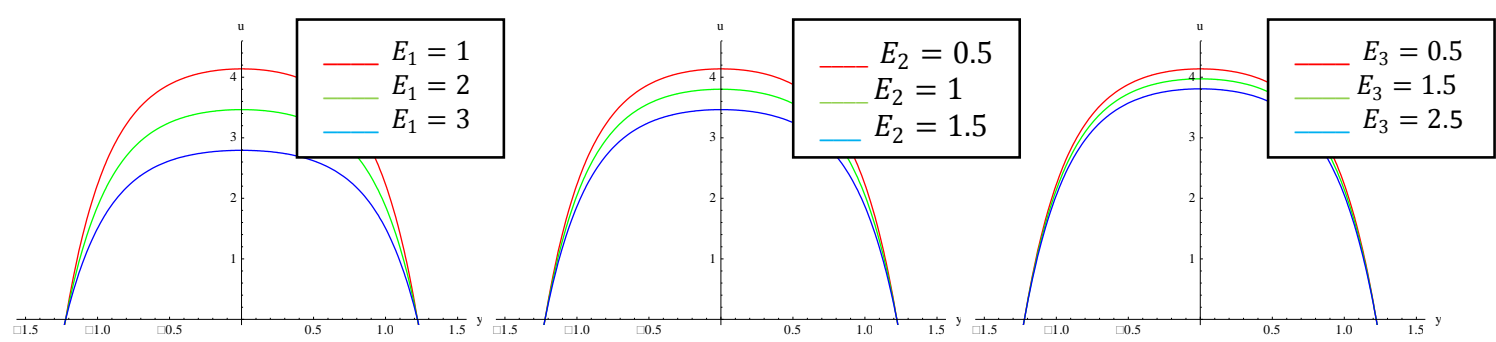

Figs. (29), (30), (31) variation of velocity $u$ for different value of $E_{1}, E_{2}, E_{3}$ respectively

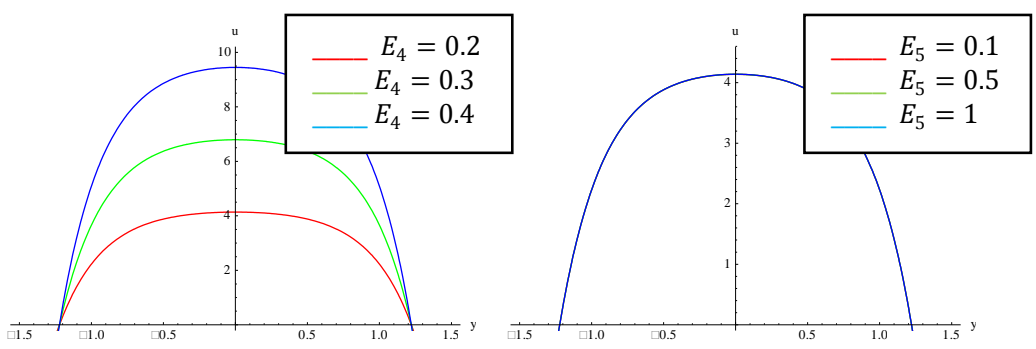

Figs. (32), (33) variation of velocity $u$ for different value of $E_{4}, E_{5}$ respectively

\section{Conclusion}

The influence of velocity slip on MHD peristaltic flow through a porous medium with heat and mass transfer is investigated. The exact solutions for stream function and temperature have been developed. The features of the flow characteristics are analyzed by plotting graphs and discussed in detail. The important findings of the present study are as follows:

1- The size of trapped bolus increases with increasing slip parameter $\beta$, permeability parameter $K$, nonuniform parameter $m$, time $t$ wall compliant parameters $E_{3}, E_{4}, E_{5}$ and $\epsilon$.

2- The size of trapped bolus decreases with increasing Hartmann number $M$ and wall compliant parameters $E_{1}, E_{2}$.

3- The temperature field increases with an increase in permeability parameter $K$, non-uniform parameter $m$, Brinkman number $B r$, wall compliant parameters $E_{3}, E_{4}$ and $\epsilon$.

4- The temperature field decreases with an increase in Hartmann number $M$, velocity slip parameter $\beta$, wall compliant parameters $E_{1}, E_{2}$ and $x$.

5- An increase in time $t$ result in increase in the temperature distribution and then decrease it.

6- The velocity field increases by increasing permeability parameter $K$, velocity slip parameter $\beta$, non-uniform parameter $m$, wall compliant parameter $E_{4}, x$ and $\epsilon$.

7- The velocity field decreases by increasing Hartmann number $M$, wall compliant parameters $E_{1}, E_{2}, E_{3}$ and time $t$.

8- The temperature and velocity field does not effected via changing $E_{5}$.

\section{Acknowledgements}

The author thanks the anonymous reviewers for their valuable comments which led to the improvement of the paper.

\section{References}

[1] T.W. Latham, Fluid motion in a peristaltic pump, MS thesis, MIT Cambridge, MA, 1966.

[2] M.Y. Jaffrin, A.H. Shapiro, Peristaltic pumping, Ann. Rev. Fluid Mech. 3 (1971) 13-16.

[3] G. Radhakrishnamacharya, V.R. Murthy, Heat transfer to peristaltic transport in a non-uniform channel, Def. Sci. 43 (1993) 275280.

[4] G. Radhakrishnamacharya, C. Srinivasulu, Influence of wall properties on peristaltic transport with heat transfer, C.R. Mec. 335 (2007) 369-373

[5] M.H. Haroun, Effect of Deborah number and phase difference on peristaltic transport of a third order fluid in an asymmetric channel, Commun. Nonlinear Sci. Numer. Simul. 12 (2007) 1464-1480.

[6] T. Hayat, M. Javed, N. Ali, MHD peristaltic transport of Jeffrey fluid in a channel with compliant walls and porous space, Transp. Porous Media 74 (2008) 259-274.

[7] K. Vajravelu, G. Radhakrishnamacharya, V. Radhakrishnamurty, Peristaltic flow and heat transfer in a vertical porous annulus with long wave approximation, Int. J. Non-Linear Mech. 42 (2007) 754-759.

[8] Kh.S. Mekheimer, Y. Abd. Elmaboud, The influence of heat transfer and magnetic field on peristaltic transport of a Newtonian fluid in a vertical annulus: application of an endoscope, Phys. Lett. A 372 (2008) 1657-1665.

[9] M. Kothandapani, S. Srinivas, On the influence of wall properties in the MHD peristaltic transport with heat transfer and porous medium, Phys. Lett. A 372 (2008) 4586-4591. 
[10] S. Nadeem, T. Hayat, N.S. Akbar, M.Y. Malik, On the influence of heat transfer in peristalsis with variable viscosity, Int. J. Heat Mass Transfer 52 (2009) 4722-4730.

[11] S. Srinivas, M. Kothandapani, The influence of heat and mass transfer on MHD peristaltic flow through a porous space with compliant walls, Appl. Math. Comput. 213 (2009) 197-208.

[12] C. Derek, D.C. Tretheway, C.D. Meinhart, Apparent fluid slip at hydrophobic micro channel walls, physics of fluids, Phys. Fluids 14 (2002) L9-L12.

[13] A. Ebaid, Effects of magnetic field and wall slip conditions on the peristaltic transport of a Newtonian fluid in an asymmetric channel, Phys. Lett. A 372 (2008) 4493-4499.

[14] T. Hayat, Q. Hussain, M. Umar Qureshi, N. Ali, Awatif.A. Hendi, Influence of slip condition on the peristaltic transport in an asymmetric channel with heat transfer: an exact solution, Int. J. Numer. Methods Fluids 67 (2011) 1944-1959. 